\title{
Equivariant K-theory, wreath products, and Heisenberg algebra
}

\author{
Weiqiang Wang*
}

\begin{abstract}
Given a finite group $G$ and a $G$-space $X$, we show that a direct sum $\mathcal{F}_{G}(X)=\bigoplus_{n \geq 0} K_{G_{n}}\left(X^{n}\right) \otimes \mathbb{C}$ admits a natural graded Hopf algebra and $\lambda$-ring structure, where $G_{n}$ denotes the wreath product $G \sim S_{n} . \mathcal{F}_{G}(X)$ is shown to be isomorphic to a certain supersymmetric product in terms of $K_{G}(X) \otimes \mathbb{C}$ as a graded algebra. We further prove that $\mathcal{F}_{G}(X)$ is isomorphic to the Fock space of an infinite dimensional Heisenberg (super)algebra. As one of several applications, we compute the orbifold Euler characteristic $e\left(X^{n}, G_{n}\right)$.
\end{abstract}

\section{Introduction}

Given a finite group $G$ and a locally compact, Hausdorff, paracompact $G$-space $X$, the $n$-th direct product $X^{n}$ admits a natural action of the wreath product $G_{n}=G \sim S_{n}$ which is a semi-direct product of the $n$-th direct product $G^{n}$ of $G$ and the symmetric group $S_{n}$. The main goal of the present paper is to study the equivariant topological K-theory $K_{G_{n}}\left(X^{n}\right)$, for all $n$ together, and discuss several applications which are of independent interest.

We will first show that a direct sum

$$
\mathcal{F}_{G}(X)=\bigoplus_{n \geq 0} K_{G_{n}}\left(X^{n}\right) \bigotimes \mathbb{C}
$$

carries several wonderful structures. More explicitly, we show that $\mathcal{F}_{G}(X)$ admits a natural Hopf algebra structure with a certain induction functor as multiplication and a certain restriction functor as comultiplication (cf. Theorem 2). When $X$ is a point, $K_{G_{n}}\left(X^{n}\right)$ is the Grothendieck ring $R\left(G_{n}\right)$, and we recover the standard Hopf algebra structure of $\bigoplus_{n \geq 0} R\left(G_{n}\right)$ (cf. e.g. [M1, M2, Z]). A key lemma used here is a straightforward generalization to equivariant K-theory of a statement in the representation theory of finite groups concerning the restriction of an induced representation to a subgroup.

*1991 Mathematics Subject Classification. Primary 19L47, 17B65. 
We show that $\mathcal{F}_{G}(X)$ is a free $\lambda$-ring generated by $K_{G}(X) \otimes \mathbb{C}$ (cf. Proposition 3). We write down explicitly the Adams operations $\varphi^{n}$ 's in $\mathcal{F}_{G}(X)$. Incidentally we also obtain an equivalent way of defining the Adams operations in $K_{G}(X) \otimes \mathbb{C}$ (not over $\mathbb{Z}$ ) by means of the wreath products, generalizing a definition by Atiyah $[\mathrm{A}$ in terms of the symmetric group in the ordinary (i.e. non-equivariant) K-theory setting. When $X$ is a point we recover the $\lambda$-ring structure of $\bigoplus_{n \geq 0} R\left(G_{n}\right)$ (cf. [M1]).

As a graded algebra $\mathcal{F}_{G}(X)$ has a simple description as a certain supersymmetric algebra in terms of $K_{G}(X) \otimes \mathbb{C}$ (cf. Theorem 3). The proof uses a theorem in [AS] and the structures of the centralizer group of an element in $G_{n}$ and of the fixed point set of the action of $a \in G_{n}$ on $X^{n}$ which we work out in Sect. 1. In particular, this description indicates that $\mathcal{F}_{G}(X)$ has the size of a Fock space of a certain infinite-dimensional Heisenberg superalgebra which we will construct in terms of natural additive maps in K-theory (cf. Theorem (1).

Our results above generalize Segal's work [S2], and our proofs are direct generalizations of those in [S2] (also see [Z, M1]). What Segal studied in [S2], partly motivated by remarks in Grojnowski Gr], is the space $\bigoplus_{n \geq 0} K_{S_{n}}\left(X^{n}\right) \otimes \mathbb{C}$ for compact $X$ which corresponds to our special case when $G$ is trivial and then $G_{n}$ is the symmetric group $S_{n}$. The paper [G] was in turn motivated by a physical paper of Vafa and Witten [VW]. Our present work grew out of an attempt to understand Segal's outlines [S2] and was also stimulated by Nakajima's lecture notes on Hilbert schemes $\mathbb{N}$ (also cf. $\mathbb{N 1}, \mathbb{G}]$ ). Our first main observation in this paper is that there is a natural way to add the group $G$ into Segal's scheme and this allows several different applications as discussed below. These applications are of independent interest on their own. We expect that there is also a natural way to incorporate $G$ into the remaining part of [S2].

Our addition of $G$ has already highly non-trivial consequences even in the case when $X$ is a point. By tensoring $\mathcal{F}_{G}(p t)$ with the group algebra of the Grothendieck ring $R(G)$, we obtain the underlying vector space for the vertex algebra associated to the lattice $R(G)[\mathrm{B}, \mathrm{FLM}]$. When $G$ is a finite subgroup of $S L_{2}(\mathbb{C})$, this will lead to a group theoretic construction of the Frenkel-Kac-Segal vertex representation of an affine Kac-Moody Lie algebra, which can be viewed as a new form of McKay correspondence [Md. Detail along these lines will be developed in a forthcoming paper.

An interesting case of our study is that $X$ is the complex plane $\mathbb{C}^{2}$ acted upon by a finite subgroup $G$ of $S L_{2}(\mathbb{C})$. Let $\widetilde{\mathbb{C}^{2} / G}$ denote the minimal resolution of singularities of $\mathbb{C}^{2} / G$ (cf. e.g. $\left.\mathbb{N}\right)$. Via the McKay correspondence [M], we show that either $K_{G_{n}}\left(\left(\mathbb{C}^{2}\right)^{n}\right) \otimes \mathbb{C}$ or $K_{S_{n}}\left(\left(\widetilde{\mathbb{C}^{2} / G}\right)^{n}\right) \otimes \mathbb{C}$ has the same dimension as the homology group of the Hilbert scheme of $n$ points on $\widetilde{\mathbb{C}^{2} / G}$ (cf. [G]). This fact has a straightforward generalization, cf. Remark 6. Our message here is that the wreath product plays an important role in the study of the Hilbert scheme of $n$ points on $\widetilde{\mathbb{C}^{2} / G}$ in exactly the way a symmetric group $S_{n}$ does for the Hilbert 
scheme of $n$ points on $\mathbb{C}^{2}$ (cf. $\left.\mathbb{N}, \widehat{B G}\right)$, which is in turn a special case of the former when $G$ is trivial. We will discuss these in more detail in another occasion.

For a smooth manifold $X$ acted upon by $G$, Dixon, Harvey, Vafa and Witten [DHVW] introduced a notion of orbifold Euler characteristic $e(X, G)$ in their study of string theory of orbifolds. We show that the orbifold Euler characteristic $e\left(X^{n}, G_{n}\right)$ is uniquely determined by $e(X, G)$ and $n$. In terms of a generating function, our formula reads (see Theorem 5):

$$
\sum_{n \geq 1} e\left(X^{n}, G_{n}\right) q^{n}=\prod_{r=1}^{\infty}\left(1-q^{r}\right)^{-e(X, G)}
$$

By putting $G=1$ and thus $e(X, G)=e(X)$, we recover a formula of HirzebruchHöfer [HH]. By using Eq. (11) and Göttsche's formula [G], we show that $X^{n} / G_{n}$ admits a resolution of singularities whose Euler characteristic coincides with the orbifold Euler characteristic $e\left(X^{n}, G_{n}\right)$ assuming that $X$ is a smooth quasi-projective surface and $X / G$ has a resolution of singularities whose Euler characteristic is $e(X, G)$.

In this paper the language of equivariant K-theory is used. We should also mention the very relevant construction of Heisenberg superalgebra on a direct sum over $n$ of the homology group $H\left(X^{[n]}\right)$ of Hilbert scheme of $n$ points on a smooth quasi-projective surface $X$, due to Nakajima [N1 and Grojnowski Gr independently. However the constructions and computations in terms of K-theory are simpler and work for more general spaces. Bezrukavnikov and Ginzburg [BG] have proposed a way to obtain a direct isomorphism from $K_{S_{n}}\left(X^{n}\right) \otimes \mathbb{C}$ to $H\left(X^{[n]}\right)$ for an algebraic surface $X$. Independently M. de Cataldo and L. Migliorini has recently established this isomorphism for complex surfaces CM.

The plan of this paper is as follows. In Sect. 11 we give a presentation of the wreath product $G_{n}$ and study its action on $X^{n}$. In Sect. 2 we construct a Hopf algebra structure on $\mathcal{F}_{G}(X)$. In Sect. 3 we give a description of $\mathcal{F}_{G}(X)$ as a graded algebra. In Sect. 4 we give a $\lambda$-ring structure on $\mathcal{F}_{G}(X)$. In Sect. 5 we construct the Heisenberg superalgebra which acts on $\mathcal{F}_{G}(X)$ irreducibly. In Sect. 6 we calculate the orbifold Euler characteristic $e\left(X^{n}, G_{n}\right)$ and study in detail the special case when $X$ is the complex plane acted upon by a finite subgroup of $S L_{2}(\mathbb{C})$. We have included some detail which are probably trivial to experts hoping that this may benefit readers with different backgrounds.

\section{The wreath product and its action on $X^{n}$}

Let $G$ be a finite group. We denote by $G_{*}$ the set of conjugacy classes of $G$ and $R(G)$ the Grothendieck ring of $G . R(G) \bigotimes_{\mathbb{Z}} \mathbb{C}$ can be identified with the ring of class functions $C(G)$ on $G$ by taking the character of a representation. Denote by $\zeta_{c}$ the order of the centralizer of an element lying in the conjugacy class $c$ in $G$. 
We define an inner product on $C(G)$ as usual:

$$
(\chi \mid \psi)=\frac{1}{|G|} \sum_{g \in G} \chi(g) \bar{\psi}(g), \quad \chi, \psi \in C(G) .
$$

Let $G^{n}=G \times \ldots \times G$ be the direct product of $n$ copies of $G$. Denote by $|G|$ the order of $G$, and by $[g]$ the conjugacy class of $g \in G$. The symmetric group $S_{n}$ acts on $G^{n}$ by permuting the $n$ factors: $s\left(g_{1}, \ldots, g_{n}\right)=\left(g_{s^{-1}(1)}, \ldots, g_{s^{-1}(n)}\right)$. The wreath product $G_{n}=G \sim S_{n}$ is defined to be the semidirect product of $G^{n}$ and $S_{n}$, namely the multiplication on $G_{n}$ is given by $(g, s)(h, t)=(g . s(h), s t)$, where $g, h \in G^{n}, s, t \in S_{n}$. Note that $G^{n}$ is a normal subgroup of $G_{n}$ by identifying $g \in G^{n}$ with $(g, 1) \in G_{n}$.

Given $a=(g, s) \in G_{n}$ where $g=\left(g_{1}, \ldots, g_{n}\right)$, we write $s \in S_{n}$ as a product of disjoint cycles: if $z=\left(i_{1}, \ldots, i_{r}\right)$ is one of them, the cycle-product $g_{i_{r}} g_{i_{r-1}} \ldots g_{i_{1}}$ of $a$ corresponding to the cycle $z$ is determined by $g$ and $z$ up to conjugacy. For each $c \in G_{*}$ and each integer $r \geq 1$, let $m_{r}(c)$ be the number of $r$-cycles in $s$ whose cycle-product lies in $c$. Denote by $\rho(c)$ the partition having $m_{r}(c)$ parts equal to $r$ $(r \geq 1)$ and denote by $\rho=(\rho(c))_{c \in G_{*}}$ the corresponding partition-valued function on $G_{*}$. Note that $\|\rho\|:=\sum_{c \in G_{*}}|\rho(c)|=\sum_{c \in G_{*}, r>1} r m_{r}(c)=n$, where $|\rho(c)|$ is the size of the partition $\rho(c)$. Thus we have defined a map from $G_{n}$ to $\mathcal{P}_{n}\left(G_{*}\right)$, the set of partition-valued function $\rho=(\rho(c))_{c \in G_{*}}$ on $G_{*}$ such that $\|\rho\|=n$. The function $\rho$ is called the type of $a=(g, s) \in G_{n}$. Denote $\mathcal{P}\left(G_{*}\right)=\sum_{n \geq 0} \mathcal{P}_{n}\left(G_{*}\right)$.

Given a partition $\lambda$ with $m_{r} r$-cycles $(r \geq 1)$, define $z_{\lambda}=\prod_{r \geq 1} r^{m_{r}} m_{r}$ !. This is the order of the centralizer in $S_{n}$ of an element of cycle-type $\lambda$. We shall denote by $l(\lambda)=\sum_{r \geq 1} m_{r}$ the length of $\lambda$. and

Given a partition-valued function $\rho \in \mathcal{P}\left(G_{*}\right)$, we define $l(\rho)=\sum_{c \in G_{*}} l(\rho(c))$

$$
Z_{\rho}=\prod_{c \in G_{*}} z_{\rho(c)} \zeta_{c}^{l(\rho(c))}
$$

Denote by $\sigma_{n}(c)$ the class function of $G_{n}$ which takes value $n \zeta_{c}$ at an $n$-cycle whose cycle-product lies in $c \in G_{*}$ and 0 otherwise. For $\rho=\left\{m_{r}(c)\right\}_{c, r} \in \mathcal{P}\left(G_{*}\right)$, we define

$$
\sigma^{\rho}=\prod_{c \in G_{*}, r \geq 1} \sigma_{r}(c)^{m_{r}(c)} .
$$

We regard $\sigma^{\rho}$ as the class function on $G_{n}$ which takes value $Z_{\rho}$ at elements of type $\rho$ (where $n=\|\rho\|)$ and 0 elsewhere.

We formulate some well-known facts below (cf. e.g. [M2]) which will be needed later.

Proposition 1 Two elements in $G_{n}$ are conjugate to each other if and only if they have the same type. The order of the centralizer in $G_{n}$ of an element of type $\rho$ is $Z_{\rho}$. 
We want to calculate the centralizer $Z_{G_{n}}(a)$ of $a \in G_{n}$. First we consider the typical case that $a$ has one $n$-cycle. As the centralizers of conjugate elements are conjugate subgroups, we may assume that $a$ is of the form $a=((g, 1, \ldots, 1), \tau)$ by Proposition 1, where $\tau=(12 \ldots n)$. Denote by $Z_{G}^{\Delta}(g)$, or $Z_{G}^{\Delta_{n}}(g)$ when it is necessary to specify $n$, the following diagonal subgroup of $G^{n}$ (and thus a subgroup of $\left.G_{n}\right)$ :

$$
Z_{G}^{\Delta}(g)=\left\{((h, \ldots, h), 1) \in G^{n} \mid h \in Z_{G}(g)\right\} .
$$

The following lemma follows from a direct computation.

Lemma 1 The centralizer $Z_{G_{n}}(a)$ of a in $G_{n}$ is equal to the product $Z_{G}^{\Delta}(g) \cdot\langle a\rangle$, where $\langle a\rangle$ is the cyclic subgroup of $G_{n}$ generated by a. Moreover, $a^{n} \in Z_{G}^{\Delta}(g)$ and $\left|Z_{G_{n}}(a)\right|=n\left|Z_{G}(g)\right|$.

Take a generic element $a=(g, s) \in G_{n}$ of type $\rho=(\rho(c))_{c \in G_{*}}$, where $\rho(c)$ has $m_{r}(c) r$-cycles $(r \geq 1)$. By Proposition 1, we may assume (by taking a conjugation if necessary) that the $m_{r}(c) r$-cycles are of the form

$$
g_{u r}(c)=\left((g, 1, \ldots, 1),\left(i_{u 1}, \ldots, i_{u r}\right)\right), 1 \leq u \leq m_{r}(c), g \in c .
$$

Denote $g_{r}(c)=((g, 1, \ldots, 1),(12 \ldots r))$. Throughout the paper, $\prod_{c, r}$ is understood as the product $\prod_{c \in G_{*}, r \geq 1}$.

Lemma 2 The centralizer $Z_{G_{n}}(a)$ of $a \in G_{n}$ is isomorphic to a direct product of the wreath products

$$
\prod_{c, r}\left(Z_{G_{r}}\left(g_{r}(c)\right) \sim S_{m_{r}(c)}\right)
$$

Furthermore $Z_{G_{r}}\left(g_{r}(c)\right)$ is isomorphic to $Z_{G}^{\Delta_{r}}(g) \cdot\left\langle g_{r}(c)\right\rangle$.

Proof. It follows from the first part of Lemma 11 that the centralizer $Z_{G_{n}}(a)$ should contain a certain subgroup naturally isomorphic to (3). By the second part of Lemma 1 we can count that the order of (3) is equal to $Z_{\rho}$. The lemma now follows by comparing with the order of $Z_{G_{n}}(a)$ given in Proposition 1 .

We will use $\star$ to denote the multiplication in $C\left(G_{n}\right)$ which corresponds to the tensor product in $R\left(G_{n}\right)$. We denote by $\underline{n}$ the trivial representation of $G_{n}$, and $\underline{1^{n}}$ the sign representation of $G_{n}$ in which $G^{n}$ acts trivially and $S_{n}$ acts by \pm 1 depending on a permutation is even or odd. By abuse of notations, we also use the same symbols to denote the corresponding characters as well. The following lemma follows easily from the definitions.

Lemma 3 1) Given $\rho, \widetilde{\rho} \in \mathcal{P}_{n}\left(G_{*}\right), \sigma^{\rho} \star \sigma^{\widetilde{\rho}}=\delta_{\rho, \widetilde{\rho}} Z_{\rho} \sigma^{\rho}$. In particular,

$$
\begin{aligned}
\underline{n} & =\sum_{\|\rho\|=n} Z_{\rho}^{-1} \sigma^{\rho}, \\
\underline{1^{n}} & =\sum_{\|\rho\|=n}(-1)^{n-l(\rho)} Z_{\rho}^{-1} \sigma^{\rho} .
\end{aligned}
$$


2) $\left(\sigma^{\rho} \mid \sigma^{\widetilde{\rho}}\right)=\delta_{\rho, \tilde{\rho}} Z_{\rho}$. In other words, $\sigma^{\rho}$ takes value $Z_{\rho}$ at the elements in $G_{n}$ of type $\rho$ and 0 elsewhere.

For a $G$-space $X$, we define an action of $G_{n}$ on $X^{n}$ as follows: given $a=$ $\left(\left(g_{1}, \ldots, g_{n}\right), s\right)$, we let

$$
\text { a. }\left(x_{1}, \ldots, x_{n}\right)=\left(g_{1} x_{s^{-1}(1)}, \ldots, g_{n} x_{s^{-1}(n)}\right)
$$

where $x_{1}, \ldots, x_{n} \in X$. Next we want to determine the fixed point set $\left(X^{n}\right)^{a}$ for $a \in G_{n}$. Let us first calculate in the typical case $a=((g, 1, \ldots, 1), \tau) \in G_{n}$. Note that the centralizer group $Z_{G}(g)$ preserves the $g$-fixed point set $X^{g}$.

Lemma 4 The fixed point set is

$$
\left(X^{n}\right)^{a}=\left\{(x, \ldots, x) \in X^{n} \mid x=g x\right\}
$$

which can be naturally identified with $X^{g}$. The action of $Z_{G_{n}}(a)$ on $\left(X^{n}\right)^{a}$ can be identified canonically with that of $Z_{G}(g)$ on $X^{g}$ together with the trivial action of the cyclic group $\langle a\rangle$ (cf. Lemma 1). Thus

$$
\left(X^{n}\right)^{a} / Z_{G_{n}}(a) \approx X^{g} / Z_{G}(g) .
$$

Proof. Let $\left(x_{1}, \ldots, x_{n}\right)$ be in the fixed point set $\left(X^{n}\right)^{a}$. By Eq. (6) we have

$$
\begin{aligned}
\left(x_{1}, x_{2}, x_{3}, \ldots, x_{n}\right) & =a \cdot\left(x_{1}, x_{2}, x_{3}, \ldots, x_{n}\right) \\
& =\left(g x_{n}, x_{1}, x_{2}, \ldots, x_{n-1}\right) .
\end{aligned}
$$

So all $x_{i}(i=1, \ldots, n)$ are equal to, say $x$, and $g x=x$. The remaining statements follow from Lemma 1.

All $Z_{G}(g)$ are conjugate and all $X^{g}$ are homeomorphic to each other for different representatives $g$ in a fixed conjugacy class $c \in G_{*}$. Also the orbit space $X^{g} / Z_{G}(g)$ can be identified with each other by conjugation for different representatives of $g$ in $c \in G_{*}$. We make a convention to denote $Z_{G}(g)\left(\operatorname{resp} . X^{g}, X^{g} / Z_{G}(g)\right)$ by $Z_{G}(c)$ (resp. $\left.X^{c}, X^{c} / Z_{G}(c)\right)$ by abuse of notations when the choice of a representative $g$ in $c$ is immaterial.

Lemma 5 Retain the notations in Lemma \&. The fixed point set $\left(X^{n}\right)^{a}$ can be naturally identified with $\prod_{c, r}\left(X^{c}\right)^{m_{r}(c)}$. Furthermore the orbit space $\left(X^{n}\right)^{a} / Z_{G_{n}}(a)$ can be naturally identified with

$$
\prod_{c, r} S^{m_{r}(c)}\left(X^{c} / Z_{G}(c)\right)
$$

where $S^{m}(\cdot)$ denotes the $m$-th symmetric product.

Proof. The first part easily follows from Lemma \&. By Lemma 2 and Lemma 4 , the action of $Z_{G_{n}}(a)$ on $\left(X^{n}\right)^{a}$ can be naturally identified with that of

$$
\prod_{c, r}\left(\left(Z_{G}^{\Delta_{r}}(g) \cdot\left\langle g_{r}(c)\right\rangle\right) \sim S_{m_{r}(c)}\right)
$$

on $\prod_{c, r}\left(X^{c}\right)^{m_{r}(c)}$ where $S_{m_{r}(c)}$ acts by permutation and $\left\langle g_{r}(c)\right\rangle$ acts on $X^{c}$ trivially. Thus the second part of the lemma follows. 


\section{The Hopf algebra structure on $\mathcal{F}_{G}(X)$}

Given a compact Hausdorff $G$-space $X$, we recall [A1, S1] that $K_{G}^{0}(X)$ is the Grothendieck group of $G$-vector bundles over $X$. One can define $K_{G}^{1}(X)$ in terms of the $K^{0}$ functor and a certain suspension operation, and one puts

$$
K_{G}(X)=K_{G}^{0}(X) \bigoplus K_{G}^{1}(X) .
$$

The tensor product of vector bundles gives rise to a multiplication on $K_{G}(X)$ which is super (i.e. $\mathbb{Z}_{2}$-graded) commutative. In this paper we will be only concerned about the free part $K(X) \otimes \mathbb{C}$, which will be denoted by $\underline{K}_{G}(X)$ subsequently. We denote by $\operatorname{dim} K_{G}^{i}(X)(i=0,1)$ the dimension of $K_{G}^{i}(X) \otimes \mathbb{C}$.

If $X$ is locally compact, Hausdorff and paracompact $G$-space, take the one-point compactification $X^{+}$with the extra point $\infty$ fixed by $G$. Then we define $K_{G}^{0}(X)$ to be the kernel of the map

$$
K_{G}^{0}\left(X^{+}\right) \longrightarrow K_{G}^{0}(\{\infty\})
$$

induced by the inclusion map $\{\infty\} \hookrightarrow X^{+}$. It is clear that this definition is equivalent to the earlier one when $X$ is compact. We also define $K_{G}^{1}(X)=K_{G}^{1}\left(X^{+}\right)$.

Note that $K_{G}(p t)$ is isomorphic to the Grothendieck ring $R(G)$ and $\underline{K}_{G}(p t)$ is isomorphic to the ring $C(G)$ of class functions on $G$. The bilinear map $\star$ induced from the tensor product

$$
K_{G}(p t) \otimes K_{G}(X) \longrightarrow K_{G}(X)
$$

gives rise to a natural $\underline{K}_{G}(p t)$-module structure on $\underline{K}_{G}(X)$. Thus $\underline{K}_{G}(X)$ naturally decomposes into a direct sum over the set of conjugacy classes $G_{*}$ of $G$. The following theorem [AS] (also cf. [B]] ) gives a description of each direct summand.

Theorem 1 There is a natural $\mathbb{Z}_{2}$-graded isomorphism

$$
\phi: \underline{K}_{G}(X) \longrightarrow \bigoplus_{[g]} \underline{K}\left(X^{g} / Z_{G}(g)\right) .
$$

Given $c \in G_{*}$, we denote by $\sigma_{c}$ the class function which takes value $\zeta_{c}$ at an element of $G$ lying in the conjugacy class $c$ and 0 otherwise. Then an element in $\underline{K}_{G}(X)$ can be written as of the form $\sum_{c \in G_{*}} \xi_{c} \sigma_{c}$, where $\xi_{c} \in \underline{K}\left(X^{g} / Z_{G}(g)\right)$. More explicitly the isomorphism $\phi$ is defined as follows when $X$ is compact: if $E$ is a $G$-vector bundle over $X$ its restriction to $X^{g}$ is acted by $g$ with base points fixed. Thus $\left.E\right|_{X^{g}}$ splits into a direct sum of subbundles $E_{\mu}$ consisting of eigenspaces of $g$ fiberwise for each eigenvalue $\mu$ of $g . Z_{G}(g)$ acts on $X^{g}$ and one may check that $\sum_{\mu} \mu E_{\mu}$ indeed lies in the $Z_{G}(g)$-invariant part of $\underline{K}^{0}\left(X^{g}\right)$. Put

$$
\phi_{g}(E)=\sum_{\mu} \mu E_{\mu} \in \underline{K}^{0}\left(X^{g} / Z_{G}(g)\right)=\underline{K}^{0}\left(X^{g}\right)^{Z_{G}(g)} .
$$


The isomorphism $\phi$ on the $K^{0}$ part is given by $\phi=\bigoplus_{[g] \in G_{*}} \phi_{g}$. Then one easily extends the isomorphism $\phi$ to $K^{1}$ as $K_{G}^{1}(X)$ can be identified with the kernel of the map from $K_{G}^{0}\left(X \times S^{1}\right)$ to $K_{G}^{0}(X)$ given by the inclusion of a point in $S^{1}$. When $X$ is a point the isomorphism $\phi$ becomes the map from a representation of $G$ to its character. By some standard arguments using compact pairs A1, the isomorphism remains valid for a locally compact, Hausdorff and paracompact $G$-space. The following lemma is well known.

Lemma 6 Given a finite group $G$, a subgroup $H$ of $G$, and a $G$-space $X$. There is a natural induction functor Ind $=\operatorname{Ind} d_{H}^{G}: K_{H}(X) \longrightarrow K_{G}(X)$. In particular when $X$ is a point, the functor $I n d_{H}^{G}$ reduces to the familiar induction functor of representations.

Proof. Note that there is a $G$-equivariant isomorphism

$$
G \times_{H} X \stackrel{\text { Iso }}{\longrightarrow} G / H \times X
$$

by sending $(g, x) \in G \times_{H} X$ to $(g H, g x)$. We remark that although both sides of (7) remain well-defined for a $H$-spcase $X$ without a $G$-action, the map Iso makes sense only for a $G$-space $X$. Denote by $p: G \times_{H} X \longrightarrow X$ the composition of the projection $G / H \times X$ to $X$ with the isomorphism (7]). As is well known [S1], one has a natural isomorphism

$$
K_{H}(X) \longrightarrow K_{G}\left(G \times_{H} X\right)
$$

by sending an $H$-equivariant vector bundle $V$ on $X$ to the $G$-equivariant vector bundle $G \times_{H} V$. The composition $p \circ \pi$ of the projection $p: G \times_{H} X \longrightarrow X$ with the bundle map $\pi: G \times_{H} V \longrightarrow G \times_{H} X$ sends $(g, v)$ to $(g, \pi(v))$. One easily check that this gives rise to a well-defined $G$-equivariant vector bundle on $X$, which induces the induction functor $\operatorname{Ind}_{H}^{G}: K_{H}(X) \longrightarrow K_{G}(X)$.

We denote by $\operatorname{Res}_{H}^{G}$ (or $\operatorname{Res}_{H}$, or even Res, if there is no ambiguity) the restriction functor from $K_{G}(X)$ to $K_{H}(X)$ by regarding a $G$-equivariant vector bundle as an $H$-equivariant vector bundle. Denote

$$
\mathcal{F}_{G}(X)=\bigoplus_{n \geq 0} \underline{K}_{G_{n}}\left(X^{n}\right), \quad \mathcal{F}_{G}^{q}(X)=\bigoplus_{n \geq 0} q^{n} \underline{K}_{G_{n}}\left(X^{n}\right)
$$

where $q$ is a formal variable counting the graded structure of $\mathcal{F}_{G}(X)$. We introduce the notion of $q$-dimension:

$$
\operatorname{dim}_{q} \mathcal{F}_{G}(X)=\sum_{n \geq 0} q^{n} \operatorname{dim} K_{G_{n}}\left(X^{n}\right)
$$

Define a multiplication $\cdot$ on $\mathcal{F}_{G}(X)$ by a composition of the induction map and the Künneth isomorphism $k$ :

$$
\underline{K}_{G_{n}}\left(X^{n}\right) \bigotimes \underline{K}_{G_{m}}\left(X^{m}\right) \stackrel{k}{\longrightarrow} \underline{K}_{G_{n} \times G_{m}}\left(X^{n+m}\right) \stackrel{\text { Ind }}{\longrightarrow} \underline{K}_{G_{n+m}}\left(X^{n+m}\right) .
$$


We denote by 1 the unit in $K_{G_{0}}\left(X^{0}\right) \cong \mathbb{C}$.

On the other hand we can define a comultiplication $\Delta$ on $\mathcal{F}_{G}(X)$, given by a composition of the inverse of the Künneth isomorphism and the restriction from $G_{n}$ to $G_{k} \times G_{n-k}$ :

$$
\underline{K}_{G_{n}}\left(X^{n}\right) \longrightarrow \bigoplus_{m=0}^{n} \underline{K}_{G_{m} \times G_{n-m}}\left(X^{n}\right) \stackrel{k^{-1}}{\longrightarrow} \bigoplus_{m=0}^{n} \underline{K}_{G_{m}}\left(X^{m}\right) \bigotimes \underline{K}_{G_{n-m}}\left(X^{n-m}\right)
$$

We define the counit $\epsilon: \mathcal{F}_{G}(X) \longrightarrow \mathbb{C}$ by sending $\underline{K}_{G_{n}}\left(X^{n}\right)(n>0)$ to 0 and $1 \in K_{G_{0}}\left(X^{0}\right) \approx \mathbb{C}$ to 1 . The antipode can be also easily defined (see Remark 2).

Theorem 2 With various operations defined as above, $\mathcal{F}_{G}(X)$ is a graded Hopf algebra.

To prove Theorem 2, we will need some preparation. Given two subgroups $H$ and $L$ of a finite group $\Gamma$ and a $\Gamma$-space $Y$, and let $V$ be an $H$-equivariant vector bundle on $Y$. We denote the action of $H$ on $V$ by $\rho$. Choose a set of representatives $S$ of the double coset $H \backslash \Gamma / L . H_{s}=s H s^{-1} \cap L$ is a subgroup of $L$ for $s \in S$. We denote by $V_{s}$ the $H_{s}$-equivariant vector bundle on $Y$ which is the same as $V$ as a vector bundle and has the conjugated action

$$
\rho^{s}(x)=\rho\left(s^{-1} x s\right), \quad x \in H_{s}
$$

Lemma $7 \operatorname{Res}_{L} \operatorname{In} d_{H}^{\Gamma} V$ is isomorphic to the direct sum of the L-equivariant vector bundles $\operatorname{Ind}_{H_{s}}^{L} V_{s}$ for all $s \in H \backslash \Gamma / L$.

One easily shows that one can extend $V$ in Lemma 7 to the whole $K_{H}(Y)$. In the case $Y=p t$, an $H$-equivariant vector bundle is just an $H$-module, and the induction and restriction functors become the more familiar ones in representation theory. In such a case, the above lemma is standard (cf. e.g. Ser], Proposition 2.2). In view of our construction of the induction functor and the restriction functor, the proof of Lemma $\square$ is essentially the same as in the case $X=p t$ which we refer to [Ser for a proof.

Proof of Theorem 8 . We will show below that the comultiplication $\Delta$ is an algebra homomorphism. The other Hopf algebra axioms are easy to check.

We apply Lemma 7 to the case $Y=X^{N}, L=G_{m} \times G_{n}, H=G_{l} \times G_{r}$, and $\Gamma=$ $G_{N}$, where $l+r=m+n=N$. In this case the double coset $H \backslash \Gamma / L$ is isomorphic to $\left(S_{l} \times S_{r}\right) \backslash S_{N} /\left(S_{m} \times S_{n}\right)$ since $G_{N}=G^{N} \cdot S_{N}$ and $G_{l} \times G_{r}=G^{N} \cdot\left(S_{l} \times S_{r}\right)$. Furthermore $\left(S_{l} \times S_{r}\right) \backslash S_{N} /\left(S_{m} \times S_{n}\right)$ is parametrized by the $2 \times 2$ matrices

$$
\left[\begin{array}{ll}
a_{11} & a_{12} \\
a_{21} & a_{22}
\end{array}\right]
$$


satisfying

$$
\begin{array}{r}
a_{i j} \in \mathbb{Z}_{+}, \quad i, j=1,2, \\
a_{11}+a_{12}=m, \quad a_{21}+a_{22}=n, \\
a_{11}+a_{21}=l, \quad a_{12}+a_{22}=r .
\end{array}
$$

We denote by $\mathcal{M}$ the set of all the $2 \times 2$ matrices of the form (10) satisfying the conditions (11).

Given $E \in \underline{K}_{G_{l}}\left(X^{l}\right), F \in \underline{K}_{G_{r}}\left(X^{r}\right)$, we calculate by using Lemma 7 as follows:

$$
\begin{aligned}
\operatorname{Res}_{(m, n)} \operatorname{Ind}_{(l, r)}^{N}(E \otimes F) & =\bigoplus_{A \in \mathcal{M}} \operatorname{Ind}_{A}^{(m, n)}\left(1_{a_{11}} \otimes T^{\left(a_{12}, a_{21}\right)} \otimes 1_{a_{22}}\right)\left(\operatorname{Res}_{A^{\prime}}(E \otimes F)\right) \\
& =\bigoplus_{A \in \mathcal{M}} \operatorname{Ind}_{\left(a_{11}, a_{12}\right)}^{m} F_{1} \otimes \operatorname{Ind}_{\left(a_{21}, a_{22}\right)}^{n} F_{2} .
\end{aligned}
$$

Here the superscript or subscript $(a, b)$ is a short-hand notations for $G_{a} \times G_{b}$. $1_{a}$ stands for the identity operator from $\underline{K}_{G_{a}}\left(X^{a}\right)$ to itself, and $\operatorname{Ind}_{(a, b)}^{a+b}$ for the induction functor from $\underline{K}_{G_{a} \times G_{b}}\left(X^{a+b}\right)$ to $\underline{K}_{G_{a+b}}\left(X^{a+b}\right)$. $T^{(a, b)}$ denotes the canonical functor from $\underline{K}_{G_{a}}\left(X^{a}\right) \otimes \underline{K}_{G_{b}}\left(X^{b}\right)$ to $\underline{K}_{G_{b}}\left(X^{b}\right) \otimes \underline{K}_{G_{a}}\left(X^{a}\right)$ by switching the factors with an appropriate sign coming from the $\mathbb{Z}_{2}$ grading of K-theory. Given $A \in \mathcal{M}$ of the form (10), the $A$ in the expressions $\operatorname{Res}_{A}$, Ind $\operatorname{Ind}_{A}$ etc stands for $G_{A} \equiv G_{a_{11}} \times$ $G_{a_{12}} \times G_{a_{21}} \times G_{a_{22}}$ while $A^{\prime}$ for $G_{A^{\prime}} \equiv G_{a_{11}} \times G_{a_{21}} \times G_{a_{12}} \times G_{a_{22}}$. We wrote $\left(1 \otimes T^{\left(a_{12}, a_{21}\right)} \otimes 1\right)\left(\operatorname{Res}_{A^{\prime}}(E \otimes F)\right)$ as $F_{1} \otimes F_{2}$ instead of a direct sum of the form $F_{1} \otimes F_{2}$ in order to simplify notations, with $F_{i}(i=1,2)$ the corresponding elements in $K_{G_{a_{i 1}} \times G_{a_{i 2}}}\left(X^{a_{11}+a_{12}}\right)$.

Now it is straightforward to check that the statement that $\Delta$ is an algebra homomorphism is just a reformulation of the identity obtained by summing Eq. (12) over all possible $(m, n)$ with $m+n=N$.

\section{A description of $\mathcal{F}_{G}(X)$ as a graded algebra}

In this section, we give an explicit description of $\mathcal{F}_{G}(X)$ as a graded algebra which in particular tells us the dimension of $\underline{K}_{G_{n}}\left(X^{n}\right)$.

Theorem 3 As a $\left(\mathbb{Z}_{+} \times \mathbb{Z}_{2}\right)$-graded algebra $\mathcal{F}_{G}^{q}(X)$ is isomorphic to the supersymmetric algebra $\mathcal{S}\left(\bigoplus_{n \geq 1} q^{n} \underline{K}_{G}(X)\right)$. In particular,

$$
\operatorname{dim}_{q} \mathcal{F}_{G}(X)=\frac{\prod_{r \geq 1}\left(1+q^{r}\right)^{\operatorname{dim} K_{G}^{1}(X)}}{\prod_{r \geq 1}\left(1-q^{r}\right)^{\operatorname{dim} K_{G}^{0}(X)}} .
$$

The supersymmetric algebra here is equal to the tensor product of the symmetric algebra $S\left(\bigoplus_{n \geq 1} q^{n} \underline{K}_{G}^{0}(X)\right)$ and the exterior algebra $\Lambda\left(\oplus_{n \geq 1} q^{n} \underline{K}_{G}^{1}(X)\right)$. 
Proof. Take $a \in G_{n}$ of type $\rho=\left\{m_{r}(c)\right\}_{c, r}$ as in Sect. 11. By Lemma 2 and Lemma 5 and the Künneth formula, we have

$$
\begin{aligned}
K\left(\left(X^{n}\right)^{a} / Z_{G_{n}}(a)\right) & \approx \bigotimes_{c \in G_{*}, r \geq 1}\left(\left(K\left(X^{c}\right)^{Z_{G}(c)}\right)^{\bigotimes m_{r}(c)}\right)^{S_{m_{r}(c)}} \\
& \approx \bigotimes_{c \in G_{*}, r \geq 1} \mathcal{S}^{m_{r}(c)}\left(K\left(X^{c} / Z_{G}(c)\right)\right) .
\end{aligned}
$$

Thus if we take a summation of Eq. (13) over all conjugacy classes of $G_{n}$ and all over $n \geq 0$, we obtain:

$$
\begin{array}{rlr}
\mathcal{F}_{G}^{q}(X) & \approx \bigoplus_{n \geq 0} \bigoplus_{\left\{m_{r}(c)\right\}_{c, r} \in \mathcal{P}_{n}\left(G_{*}\right)} q^{n} \bigotimes_{c, r} \mathcal{S}^{m_{r}(c)}\left(\underline{K}\left(X^{c} / Z_{G}(c)\right)\right) & \text { by Theorem } \mathbb{1}, \\
& =\bigoplus_{\left\{m_{r}(c)\right\}_{c, r} \in \mathcal{P}\left(G_{*}\right)} \bigotimes_{c, r} \mathcal{S}^{m_{r}(c)}\left(q^{r} \underline{K}\left(X^{c} / Z_{G}(c)\right)\right) & \\
& =\bigoplus_{\left\{m_{r}\right\}_{r}} \bigotimes_{r \geq 1} \mathcal{S}^{m_{r}}\left(\bigoplus_{c \in G_{*}} q^{r} \underline{K}\left(X^{c} / Z_{G}(c)\right)\right) & \text { by letting } m_{r}=\sum_{c \in G_{*}} m_{r}(c), \\
& =\bigoplus_{\left\{m_{r}\right\}_{r}} \bigotimes_{r \geq 1} \mathcal{S}^{m_{r}}\left(q^{r} \underline{K}_{G}(X)\right) & \text { by Theorem } \mathbb{1}, \\
& =\mathcal{S}\left(\bigoplus_{r \geq 1} q^{r} \underline{K}_{G}(X)\right) .
\end{array}
$$

The statement concerning $\operatorname{dim}_{q} \mathcal{F}_{G}(X)$ is an immediate consequence.

Remark 1 1) Theorem 3 in the case when $G$ is the trivial group (and so $G_{n}=$ $\left.S_{n}\right)$ is due to Segal [S2]. Our proof is adapted from his to the wreath product setting.

2) If $G$ acts on $X$ freely, so does $G^{n}$ on $X^{n}$. Then we have the isomorphism $K(X / G) \approx K_{G}(X)$. Note that $G^{n}$ is a normal subgroup of the wreath product $G_{n}$ and the quotient $G_{n} / G^{n}$ is isomorphic to $S_{n}$. By Proposition 2.1 in Segal [S1] we see that

$$
K_{G_{n}}\left(X^{n}\right) \approx K_{G_{n} / G^{n}}\left(X^{n} / G^{n}\right)=K_{S_{n}}\left((X / G)^{n}\right) .
$$

Therefore Theorem 3 follows from the special case $G=1$ of Theorem 3 (applying to $X / G$ ) and Eq. (14).

3) When $X$ is a point, $\mathcal{F}_{G}(p t)=\bigoplus_{n \geq 0} R\left(G_{n}\right)$, and $\sigma^{\rho}, \rho \in \mathcal{P}\left(G_{*}\right)$ form a linear basis for $\mathcal{F}_{G}(p t)$ (cf. [M2]). In particular,

$$
\operatorname{dim}_{q} \mathcal{F}_{G}(p t)=\prod_{r \geq 1}\left(1-q^{r}\right)^{-\left|G_{*}\right|}
$$


4) One may reformulate Theorem 3 in terms of the de-localized equivariant cohomology $\mathrm{BBM}, \mathrm{BC}$ via the Chern character.

Remark 2 The Hopf algebra defined in Sect. 2 can be identified via the isomorphism in Theorem 3 with the standard one on the supersymmetric algebra $\mathcal{S}\left(\oplus_{n \geq 1} \underline{K}_{G}(X)[n]\right)$ by showing the sets of primitive vectors correspond to each other. Here $\underline{K}_{G}(X)[n]$ denotes the $n$-th copy of $\underline{K}_{G}(X)$ (see Theorem 3 ). The antipode of the former space can be transfered via the isomorphism from the latter one.

\section{The $\lambda$-ring structure on $\mathcal{F}_{G}(X)$}

Let us denote by $c_{n}$ the conjugacy class in $G_{n}$ which has the type of an $n$-cycle and whose cycle product lies in the conjugacy class $c \in G_{*}$. We consider the following diagram of K-theory maps:

$$
\begin{aligned}
\underline{K}_{G}(X) \stackrel{\otimes n}{\longrightarrow} & \underline{K}_{G_{n}}\left(X^{n}\right) \stackrel{\phi_{n}}{\longrightarrow} \bigoplus_{[a] \in\left(G_{n}\right)_{*}} \underline{K}\left(\left(X^{n}\right)^{a} / Z_{G_{n}}(a)\right) \\
& \stackrel{p r}{\rightleftarrows} \\
& \bigoplus_{c \in G_{*}} \underline{K}\left(\left(X^{n}\right)^{c_{n}} / Z_{G_{n}}\left(c_{n}\right)\right) \stackrel{\vartheta}{\longrightarrow} \bigoplus_{c \in G_{*}} \underline{K}\left(X^{c} / Z_{G}(c)\right) \\
\stackrel{\phi}{\longleftarrow} & \underline{K}_{G}(X) .
\end{aligned}
$$

Given a $G$-equivariant vector bundle $V$, we define a $G_{n}$-action on the $n$-th outer tensor product $V^{\otimes n}$ by letting

$$
\left(\left(g_{1}, \ldots, g_{n}\right), s\right) \cdot v_{1} \otimes \ldots \otimes v_{n}=g_{1} v_{s^{-1}(1)} \otimes \ldots \otimes g_{n} v_{s^{-1}(n)}
$$

where $g_{1}, \ldots, g_{n} \in G, s \in S_{n}$. Clearly $V^{\bigotimes n}$ endowed with such a $G_{n}$ action is a $G_{n^{-}}$ equivariant vector bundle over $X^{n}$. Sending $V$ to $V^{\bigotimes n}$ gives rise to the K-theory map $\nabla n$. $\phi_{n}$ is the isomorphism in Theorem 1 when applying to the case $X^{n}$ with the action of $G_{n}$. pr is the projection to the direct sum over the conjugacy classes of $G_{n}$ which are of the type of an $n$-cycle while $\iota$ denotes the inclusion map. $\vartheta$ denotes the natural identification given by Lemma 5 . Finally the last map $\phi$ is the isomorphism given in Theorem 1 .

We shall now define a $\lambda$-ring structure on $\mathcal{F}_{G}(X)$. It suffices to define the Adams operations $\phi^{n}$ on $\mathcal{F}_{G}(X)$. We also introduce several other K-theory operations which will be needed later.

Definition 1 We define the following composition maps:

$$
\begin{aligned}
\psi^{n} & :=n \phi^{-1} \circ \vartheta \circ p r \circ \phi_{n} \circ \nabla n: \underline{K}_{G}(X) \longrightarrow \underline{K}_{G}(X), \\
\varphi^{n} & :=n \phi_{n}^{-1} \circ \iota \circ p r \circ \phi_{n} \circ \nabla n: \underline{K}_{G}(X) \longrightarrow \underline{K}_{G_{n}}\left(X^{n}\right), \\
c h_{n} & :=\phi^{-1} \circ \vartheta \circ p r \circ \phi_{n}: \underline{K}_{G_{n}}\left(X^{n}\right) \longrightarrow \underline{K}_{G}(X), \\
\omega_{n} & :=n \phi_{n}^{-1} \circ \iota \circ \vartheta^{-1} \circ \phi: \underline{K}_{G}(X) \longrightarrow \underline{K}_{G_{n}}\left(X^{n}\right) .
\end{aligned}
$$


We list some properties of these K-theory maps whose proof is straightforward.

Proposition 2 The following identities hold:

$$
c h_{n} \circ \omega_{n}=n I d, \quad \omega_{n} \circ \psi^{n}=n \varphi^{n}, \quad c h_{n} \circ \varphi^{n}=n \psi^{n} .
$$

Recall that $\sigma_{n}(c)$ is the class function of $G_{n}$ which takes value $n \zeta_{c}$ at an $n$-cycle whose cycle-product lies in $c \in G_{*}$ and 0 otherwise.

Lemma 8 1) $\varphi^{n}(V)=\sum_{c \in G_{*}} \zeta_{c}^{-1} V^{\bigotimes n} \star \sigma_{n}(c)$.

2) Both $\psi^{n}$ and $\varphi^{n}$ are additive K-theory maps.

Note that the order of the centralizer of an element lying in the conjugacy class $c_{n}$ is equal to $n \zeta_{c}$. The first part of the above lemma now follows from the definition of $\varphi^{n}$ and Lemma 3. The second part can be proved in exactly the same way as in the symmetric group case [A]. We record here only a useful formula in the proof: Given $V, W$ two $G$-equivariant vector bundles, let $[V]$ denote the corresponding element of $V$ in $K_{G}(X)$. (In general we use $V$ itself to denote the corresponding element in $K_{G}(X)$ by abuse of notation). Then

$$
([V]-[W])^{\bigotimes n}=\sum_{j=0}^{n}(-1)^{j} \operatorname{Ind}_{G_{n-j} \times G_{j}}^{G_{n}}\left[V^{\bigotimes(n-j)} \otimes W^{\bigotimes j}\right] \in K_{G_{n}}\left(X^{n}\right) .
$$

Here $V^{\otimes(n-j)}$ endows the standard $G_{n-j}$-action given by substituting $n$ with $n-j$ in Eq. (15), and $G_{j}$ acts on $W^{\bigotimes j}$ by the tensor product of the standard $G_{j}$-action with the sign representation of $G_{j}$.

$\psi^{n}$ 's are the Adams operations on $\underline{K}_{G}(X)$, giving rise to the $\lambda$-ring structure on $\underline{K}_{G}(X)$. Theorem 3 ensures us that $\mathcal{F}_{G}(X)$ as a $\lambda$-ring is free and generated by $\underline{K}_{G}(X)$.

Proposition $3 \mathcal{F}_{G}(X)$ is a free $\lambda$-ring generated by $K_{G}(X) \otimes \mathbb{C}$, with $\varphi^{n}$ 's as the Adams operations.

Remark 3 If $X$ is a point, then $K_{G_{n}}(p t)=R\left(G_{n}\right)$ and $\mathcal{F}_{G}(p t)=\bigoplus_{n \geq 0} R\left(G_{n}\right)$. Our result reduces to the fact that $\mathcal{F}_{G}(p t)$ is a free $\lambda$-ring generated by $G_{*}$ [M1]. In the case when $G=1$, the proposition was due to Segal [52].

Denote by $\widehat{\mathcal{F}}_{G}^{q}(X)$ the completion of $\mathcal{F}_{G}^{q}(X)$ which allows formal infinite sums. Given $V \in \underline{K}_{G}(X)$, we introduce $H(V, q), E(V, q) \in \widehat{\mathcal{F}}_{G}^{q}(X)$ as follows:

$$
\begin{aligned}
& H(V, q)=\bigoplus_{n \geq 0} q^{n} V^{\otimes n}, \\
& E(V, q)=\bigoplus_{n \geq 0} q^{n} V^{\otimes n} \star \underline{1^{n}} .
\end{aligned}
$$


Proposition 4 One can express $H(V, q)$ and $E(V, q)$ in terms of $\varphi^{r}(V)$ as follows:

$$
\begin{aligned}
H(V, q) & =\exp \left(\sum_{r>0} \frac{1}{r} \varphi^{r}(V) q^{r}\right), \\
E(V,-q) & =\exp \left(-\sum_{r>0} \frac{1}{r} \varphi^{r}(V) q^{r}\right) .
\end{aligned}
$$

Proof. We shall prove Eq. (17) by using Eq. (4). The formula for $E(V,-q)$ can be similarly obtained by using Eq. (5).

$$
\begin{aligned}
H(V, q) & =\bigoplus_{n \geq 0} q^{n} V^{\otimes n} \star \underline{n} \\
& =\bigoplus_{n \geq 0} q^{n} V^{\otimes n} \star\left(\sum_{\|\rho\|=n} Z_{\rho}^{-1} \sigma^{\rho}\right) \\
& =\bigoplus_{n \geq 0} \sum_{\|\rho\|=n}\left(Z_{\rho}^{-1} q^{n} V^{\otimes n} \star \sigma^{\rho}\right) \\
& \stackrel{(\star)}{=} \prod_{c \in G_{*}, r \geq 1} \frac{1}{m_{r}(c) !}\left(\frac{1}{r} q^{r} \zeta_{c}^{-1} V^{\otimes r} \star \sigma_{r}(c)\right)^{m_{r}(c)} \\
& =\exp \left(\sum_{r \geq 1} \frac{1}{r} q^{r} \sum_{c \in G_{*}} \zeta_{c}^{-1} V^{\bowtie r} \star \sigma_{r}(c)\right) \quad \text { by Lemma 8, } \\
& =\exp \left(\sum_{r \geq 1} \frac{1}{r} q^{r} \varphi^{r}(V)\right) .
\end{aligned}
$$

Here the equation $(\star)$ is understood by means of the multiplication in $\mathcal{F}_{G}(X)$ given by the composition (8).

Corollary 1 The $\lambda$-operations $\lambda^{n}$ on the $\lambda$ ring $\mathcal{F}_{G}(X)$ sends $V \in \underline{K}_{G}(X)$ to $V^{\otimes n} \star \underline{1^{n}}$.

Combining with the additivity of $\varphi^{r}$, we have the following.

Corollary 2 The following equations hold for $V, W \in \underline{K}_{G}(X)$ :

$$
\begin{aligned}
H(-V, q) & =E(V,-q) \\
H(V \bigoplus W, q) & =H(V, q) H(W, q) .
\end{aligned}
$$

\section{$5 \quad \mathcal{F}_{G}(X)$ and a Heisenberg superalgebra}

We see from Theorem 3 that $\mathcal{F}_{G}(X)$ has the same size of the tensor product of the Fock space of an infinite-dimensional Heisenberg algebra of rank $\operatorname{dim} K_{G}^{0}(X)$ and 
that of an infinite-dimensional Clifford algebra of $\operatorname{rank} \operatorname{dim} K_{G}^{1}(X)$. It is our next step to actually construct such an Heisenberg/Clifford algebra. We will simply refer to as Heisenberg superalgebra from now on.

The dual of $\underline{K}_{G}(X)$, denoted by $\underline{K}_{G}(X)^{*}$, is naturally $\mathbb{Z}_{2}$-graded as identified with $\underline{K}_{G}^{0}(X)^{*} \oplus \underline{K}_{G}^{1}(X)^{*}$. Denote by $\langle\cdot, \cdot\rangle$ the pairing between $\underline{K}_{G}(X)^{*}$ and $\underline{K}_{G}(X)$. For any $n, m \geq 1$ and $\eta \in \underline{K}_{G}(X)^{*}$, we define an additive map

$$
a_{-m}(\eta): \underline{K}_{G_{n}}\left(X^{n}\right) \longrightarrow \underline{K}_{G_{n-m}}\left(X^{n-m}\right)
$$

as the composition

$$
\begin{aligned}
\underline{K}_{G_{n}}\left(X^{n}\right) \stackrel{\operatorname{Res}}{\longrightarrow} \underline{K}_{G_{m} \times G_{n-m}}\left(X^{n}\right) \stackrel{k^{-1}}{\longrightarrow} \underline{K}_{G_{m}}\left(X^{m}\right) \otimes \underline{K}_{G_{n-m}}\left(X^{n-m}\right) \\
\stackrel{c h_{m} \otimes 1}{\longrightarrow} \underline{K}_{G}(X) \otimes \underline{K}_{G_{n-m}}\left(X^{n-m}\right) \stackrel{\eta \otimes 1}{\longrightarrow} \underline{K}_{G_{n-m}}\left(X^{n-m}\right) .
\end{aligned}
$$

On the other hand, we define for any $m \geq 1$ and $V \in \underline{K}_{G}(X)$ an additive map

$$
a_{m}(V): \underline{K}_{G_{n-m}}\left(X^{n-m}\right) \longrightarrow \underline{K}_{G_{n}}\left(X^{n}\right)
$$

as the composition

$$
\begin{aligned}
\underline{K}_{G_{n-m}}\left(X^{n-m}\right) & \stackrel{\omega_{m}(V) \otimes}{\longrightarrow} \\
& \stackrel{k}{\longrightarrow} \quad \underline{K}_{G_{m}}\left(X^{m}\right) \otimes \underline{K}_{G_{m} \times G_{n-m}}\left(X^{n}\right) \stackrel{\text { Ind }}{\longrightarrow} \underline{K}_{G_{n}}\left(X^{n}\right) .
\end{aligned}
$$

Let $\mathcal{H}$ be the linear span of the operators $a_{-m}(\eta), a_{m}(V), m \geq 1, \eta \in \underline{K}_{G}(X)^{*}$, $V \in \underline{K}_{G}(X)$. Clearly $\mathcal{H}$ admits a natural $\mathbb{Z}_{2}$-gradation induced from that on $\underline{K}_{G}(X)$ and $\underline{K}_{G}(X)^{*}$. Below we shall use $[\cdot, \cdot]$ to denote the supercommutator as well. It is understood that $[a, b]$ is the anti-commutator $a b+b a$ when $a, b \in \mathcal{H}$ are both odd elements according to the $\mathbb{Z}_{2}$-gradation.

Theorem 4 When acting on $\mathcal{F}_{G}(X), \mathcal{H}$ satisfies the Heisenberg superalgebra commutation relations, namely for $m, l \geq 1, \eta, \eta^{\prime} \in \underline{K}_{G}(X)^{*}, V, W \in \underline{K}_{G}(X)$,

$$
\begin{aligned}
{\left[a_{-m}(\eta), a_{l}(V)\right] } & =l \delta_{m, l}\langle\eta, V\rangle, \\
{\left[a_{m}(W), a_{l}(V)\right] } & =0 \\
{\left[a_{-m}(\eta), a_{-l}\left(\eta^{\prime}\right)\right] } & =0 .
\end{aligned}
$$

Furthermore $\mathcal{F}_{G}(X)$ is an irreducible representation of the Heisenberg superalgebra.

Proof. We may assume that $V, \eta$ are homogeneous, say of degree $v$ and $e$ where $v, e \in\{0,1\}$, according to the $\mathbb{Z}_{2}$-grading of $\underline{K}_{G}(X)$ and its dual. We keep using the notations in the proof of Theorem 2 . 
Given $E \in \underline{K}_{G_{r}}\left(X^{r}\right)$, we first observe by the definitions (18) and (19) that $a_{-m}(\eta) a_{l}(V) E$ (resp. $\left.(-1)^{v e} a_{l}(V) a_{-m}(\eta) E\right)$ is given by the composition from the top to the bottom along the left (resp. right) side of the diagram below:

$$
\begin{aligned}
& \underline{K}_{G_{r}}\left(X^{r}\right) \\
& \downarrow \omega_{l}(V) \otimes \cdot \\
& \underline{K}_{G_{l}}\left(X^{l}\right) \otimes \underline{K}_{G_{r}}\left(X^{r}\right) \\
& \begin{array}{c}
\quad \operatorname{Ind} \swarrow \\
\underline{K}_{G_{N}}\left(X^{N}\right) \\
\| \\
\underline{K}_{G_{N}}\left(X^{N}\right) \\
\operatorname{Res} \searrow
\end{array} \\
& \underline{K}_{G_{m}}\left(X^{m}\right) \otimes \underline{K}_{G_{n}}\left(X^{n}\right) \\
& \searrow 1 \otimes \operatorname{Res} \\
& \underline{K}_{G_{l}}\left(X^{l}\right) \otimes \underline{K}_{G_{m}}\left(X^{m}\right) \otimes \underline{K}_{G_{r-m}}\left(X^{r-m}\right) \\
& \downarrow T^{(l, m)} \otimes 1 \\
& \underline{K}_{G_{m}}\left(X^{m}\right) \otimes \underline{K}_{G_{l}}\left(X^{l}\right) \otimes \underline{K}_{G_{r-m}}\left(X^{r-m}\right) \\
& P \swarrow \text { Ind } \otimes 1 \\
& \downarrow c h_{m} \otimes 1 \\
& \underline{K}_{G}(X) \otimes \underline{K}_{G_{n}}\left(X^{n}\right) \\
& \downarrow \eta \otimes 1 \\
& \underline{K}_{G_{n}}\left(X^{n}\right)
\end{aligned}
$$

Here and below it is understood that when a negative integer appears in indices the corresponding term is 0 . To simplify notations, we put Res (resp. Ind) instead of the composition $k^{-1} \circ$ Res (resp. Ind $\circ k$ ) in the above diagram and below.

We denote by $\mathcal{M}^{\prime}$ the set of all the $2 \times 2$ matrices of the form (10) satisfying (11) except the following two matrices

$$
\left[\begin{array}{cc}
0 & m \\
l & r-m
\end{array}\right], \quad\left[\begin{array}{cc}
m & 0 \\
l-m & r
\end{array}\right]
$$

As in the proof of Theorem 2, we apply Lemma 7 to the case $Y=X^{N}, \Gamma=$ $G_{N}, H=G_{l} \times G_{r}$, and $L=G_{m} \times G_{n}$, where $l+r=m+n=N$.

$$
\begin{aligned}
& \operatorname{Res}_{(m, n)} \operatorname{Ind}_{(l, r)}^{N}\left(\omega_{l}(V) \otimes E\right) \\
= & \bigoplus_{A \in \mathcal{M}} \operatorname{Ind}_{A}^{(m, n)}\left(1_{a_{11}} \otimes T^{\left(a_{12}, a_{21}\right)} \otimes 1_{a_{22}}\right)\left(\operatorname{Res}_{A^{\prime}}\left(\omega_{l}(V) \otimes E\right)\right) \\
= & \bigoplus_{A \in \mathcal{M}} \operatorname{Ind}_{\left(a_{11}, a_{12}\right)}^{m} F_{1} \otimes \operatorname{Ind}_{\left(a_{21}, a_{22}\right)}^{n} F_{2} \\
= & \bigoplus_{A \in \mathcal{M}^{\prime}} \operatorname{Ind}_{\left(a_{11}, a_{12}\right)}^{m} F_{1} \otimes \operatorname{Ind}_{\left(a_{21}, a_{22}\right)}^{n} F_{2} \\
= & \bigoplus_{A \in \mathcal{M}^{\prime}} F_{1} \otimes \operatorname{Ind}_{(l, r-m)}^{n} F_{2} \bigoplus F_{1} \otimes \operatorname{Ind}_{(l-m, r)}^{n} F_{2} \\
& \left.\bigoplus 1_{m} \otimes \operatorname{Ind}_{(l, r-m)}^{n}\right)\left(T^{(l, m)} \otimes 1_{l-m}\right)\left(1_{l} \otimes \operatorname{Res}_{(m, r-m)}\right)\left(\omega_{l}(V) \otimes E\right) \\
& \left.\bigoplus\left(1_{m}^{n} \otimes \operatorname{Ind}_{(l-m, r)}^{n}\right)\right)\left(\operatorname{Res}_{(m, l-m)} \otimes 1_{r}\right)\left(\omega_{l}(V) \otimes E\right) .
\end{aligned}
$$


We get 0 when applying the map $c h_{m} \otimes 1$ to $\operatorname{Ind}_{\left(a_{11}, a_{12}\right)}^{m} F_{1} \otimes \operatorname{Ind}_{\left(a_{21}, a_{22}\right)}^{n} F_{2}$ for $A \in \mathcal{M}^{\prime}$ in (23) by Lemma 3. When applying $(\eta \otimes 1) \circ\left(c h_{m} \otimes 1\right)$ to the second term of the r.h.s. of (23), we obtain $(-1)^{v e} a_{l}(V) a_{-m}(\eta) E$. When applying $c h_{m} \otimes 1$ to the third term of the r.h.s. of (23), we get 0 if $m \neq l$ by Lemma 3 . In the case $m=l$, the third term of the r.h.s. of (23) is simply $\omega_{l}(V) \otimes E$. When applying $(\eta \otimes 1) \circ\left(c h_{m} \otimes 1\right)$ to it, we get $l\langle\eta, V\rangle E$ by Proposition \&. Putting all these pieces together, we have proved Eq. (20).

We may assume that $W$ is homogeneous of degree $w \in\{0,1\}$ according to the $\mathbb{Z}_{2}$-grading of $\underline{K}_{G}(X)$. Eq. (21) is a consequence of the transitivity of the induction functor:

$$
\begin{aligned}
& \operatorname{Ind}_{G_{m} \times G_{l+r}}^{G_{m+l}}\left(\omega_{m}(W) \otimes \operatorname{Ind}_{G_{l} \times G_{r}}^{G_{l+r}}\left(\omega_{l}(V) \otimes E\right)\right) \\
= & \operatorname{Ind}_{G_{m} \times G_{l+r}}^{G_{m}}\left(\operatorname{Ind}_{G_{m} \times G_{l} \times G_{r}}^{G_{m} \times G_{l+r}}\left(\omega_{m}(W) \otimes \omega_{l}(V) \otimes E\right)\right) \\
= & \operatorname{Ind}_{G_{m} \times G_{l} \times G_{r}}^{G_{m+l}}\left(\omega_{m}(W) \otimes \omega_{l}(V) \otimes E\right) \\
= & (-1)^{v w} \operatorname{Ind}_{G_{l} \times G_{m+r}}^{G_{m+l+r}}\left(\operatorname{Ind}_{G_{l} \times G_{m} \times G_{m}}^{G_{m} \times G_{m}}\left(\omega_{l}(V) \otimes \omega_{m}(W) \otimes E\right)\right) \\
= & \operatorname{Ind}_{G_{l} \times G_{m+r}}^{G_{m+l+r}}\left(\omega_{l}(V) \otimes \operatorname{Ind}_{G_{m} \times G_{r}}^{G_{m+r}}\left(\omega_{m}(W) \otimes E\right)\right) .
\end{aligned}
$$

Similarly Eq. (22) is a consequence of the transitivity of the restriction functor. The irreducibility of $\mathcal{F}_{G}(X)$ as a representation of $\mathcal{H}$ follows immediately from the $q$-dimension formula for $\mathcal{F}_{G}(X)$ given in Theorem of.

In the special case $G=1$, the Heisenberg superalgebra was constructed by Segal [S2] which differs slightly from ours. Our proof follows his strategy of proof as well.

Remark 4 One may consider the enlarged space

$$
V_{G}:=\mathcal{F}_{G}(p t) \otimes \mathbb{C}[R(G)]
$$

where $\mathbb{C}[R(G)]$ is the group algebra of the lattice $R(G)$. Note that $V_{G}$ is the underlying space for a lattice vertex algebra $[\mathrm{B}$, FLM]. In particular, when $G$ is a finite subgroup of $S L_{2}(\mathbb{C})$, the space $V_{G}$ is closely related to the Frenkel-Kac-Segal vertex representation of an affine Lie algebra. In this way, we are able to obtain a new link between the subgroups of $S L_{2}(\mathbb{C})$ and the affine Lie algebras widely known as the McKay correspondence. Connections among symmetric functions, $\mathcal{F}_{G}(p t), V_{G}$, and vertex operators will be developed in a forthcoming paper.

More generally, one may consider

$$
V_{G}(X):=\mathcal{F}_{G}(X) \otimes \mathbb{C}\left[K_{G}(X)\right]
$$

when $K_{G}(X)$ is torsion-free, where $\mathbb{C}\left[K_{G}(X)\right]$ is the group algebra of the lattice $\mathbb{C}\left[K_{G}(X)\right]$ (if $K_{G}(X)$ is not torsion-free we replace $K_{G}(X)$ in (24) by the free part of $K_{G}(X)$ over $\left.\mathbb{Z}\right)$. 


\section{The orbifold Euler characteristic $e\left(X^{n}, G_{n}\right)$}

In the study of string theory on orbifolds, Dixon et al [DHVW] came up with a notion of orbifold Euler characteristic defined as follows:

$$
e(X, G)=\frac{1}{|G|} \sum_{g_{1} g_{2}=g_{2} g_{1}} e\left(X^{g_{1}, g_{2}}\right),
$$

where $X$ is a smooth $G$-manifold. $X^{g_{1}, g_{2}}$ denotes the common fixed point set of $g_{1}$ and $g_{2}$ and $e(\cdot)$ denotes the usual Euler characteristic. One easily shows [HH] that the orbifold Euler characteristic can be equivalently defined as

$$
e(X, G)=\sum_{[g] \in G_{*}} e\left(X^{g} / Z_{G}(g)\right) .
$$

Denote by $X^{(n)}$ the $n$-th symmetric product of $X$. Recall that Macdonald's formula $\mathbb{M}$ relates $e\left(X^{(n)}\right)$ to $e(X)$ as follows:

$$
\sum_{n=0} e\left(X^{(n)}\right) q^{n}=(1-q)^{-e(X)} .
$$

The following theorem relates the orbifold Euler characteristic $e\left(X^{n}, G_{n}\right)$ to $e(X, G)$.

Theorem 5 We have $\sum_{n \geq 0} e\left(X^{n}, G_{n}\right) q^{n}=\prod_{r=1}^{\infty}\left(1-q^{r}\right)^{-e(X, G)}$.

Proof. For an alternative proof see Remark 5 below. By the definition of the orbifold Euler characteristic, Lemmas 2 and Lemma 5, we have

$$
\begin{array}{rlrl}
\sum_{n \geq 0} e\left(X^{n}, G_{n}\right) q^{n} & =\sum_{n \geq 0} \sum_{[a] \in\left(G_{n}\right)_{*}} e\left(\left(X^{n}\right)^{a} / Z_{G_{n}}(a)\right) q^{n} & \text { by Eq. (25), } \\
& \stackrel{(A)}{=} \sum_{n \geq 0} \sum_{\sum_{r} r m_{r}(c)=n} \prod_{c \in G_{*}} e\left(\left(X^{c} / Z_{G}(c)\right)^{m_{r}(c)}\right) q^{n} & \\
& =\prod_{c \in G_{*}} \prod_{r \geq 1}\left(\sum_{m_{r}(c) \geq 0} e\left(\left(X^{c} / Z_{G}(c)\right)^{m_{r}(c)}\right) q^{m_{r}(c)}\right) \\
& =\prod_{c \in G_{*}} \prod_{r \geq 1}\left(1-q^{r}\right)^{-e\left(X^{c} / Z_{G}(c)\right)} \quad \text { by applying (26) to } X^{c} / Z_{G}(c), \\
& =\frac{1}{\prod_{r=0}^{\infty}\left(1-q^{r}\right)^{e(X, G)}} \quad \text { by Eq. (25). }
\end{array}
$$

Here Eq. (A) follows from Lemma 2 and Lemma 5.

In the case when $G$ is trivial, we recover a formula given in [HH].

Remark 5 According to Atiyah and Segal [AS] the orbifold Euler characteristic can be calculated in terms of equivariant K-theory:

$$
e(X, G)=\operatorname{dim} K_{G}^{0}(X)-\operatorname{dim} K_{G}^{1}(X) .
$$

Theorem 5 follows from Theorem 3 by applying Eq. (27) to $K_{G_{n}}\left(X^{n}\right)$. 
One is interested [DHVW, $\overline{\mathrm{HH}}$ ] in finding a resolution of singularities

$$
\widetilde{X / G} \longrightarrow X / G
$$

with the property

$$
e(X, G)=e(\widetilde{X / G}) .
$$

We assume that $X$ is a smooth quasi-projective surface with such a property in the following discussions. Denote by $X^{[n]}$ the Hilbert scheme of $n$ points on $X$. According to Göttsche $\llbracket \mathbb{G}$, the Euler characteristic of $X^{[n]}$ is given by:

$$
\sum_{n \geq 0} e\left(X^{[n]}\right) q^{n}=\prod_{r=0}^{\infty}\left(1-q^{r}\right)^{-e(X)} .
$$

We note that $X^{n} / G_{n}$ is naturally identified with $(X / G)^{n} / S_{n}$. The following commutative diagram

$$
\begin{aligned}
\widetilde{X / G}^{[n]} & \rightarrow(\widetilde{X / G})^{n} / S_{n} \\
\downarrow & \downarrow \\
X^{n} / G_{n} & \equiv(X / G)^{n} / S_{n}
\end{aligned}
$$

implies that the Hilbert scheme $\widetilde{X / G}^{[n]}$ is a resolution of singularity of $X^{n} / G_{n}$ (indeed it is semismall). By applying Eq. (28) to $\widetilde{X / G}$ and comparing with Theorem 5 , we have the following corollary.

Corollary 3 Let $X$ be a smooth quasi-projective surface and assume that there exists a smooth resolution $\widetilde{X / G}$ of singularities of the orbifold $X / G$ such that $e(\widetilde{X / G})=e(X, G)$. Then there exists a resolution of singularities of $X^{n} / G_{n}$ given by $\widetilde{X / G}^{[n]}$ satisfying

$$
e\left(X^{n}, G_{n}\right)=e\left(\widetilde{X / G}^{[n]}\right) .
$$

The assumption of the existence of the resolution $\widetilde{X / G}$ of singularities of $X / G$ above is necessary as this is the special case of $X^{n} / G_{n}$ for $n=1$. In the setting of Corollary B, we conjecture that $\widetilde{X / G}^{[n]}$ is a crepant resolution of $X^{n} / G_{n}$ provided that $\widetilde{X / G}$ is a crepant resolution of singularities of $X / G$.

We consider a special case in detail. Let $X$ be the complex plane $\mathbb{C}^{2}$ acted upon by a finite subgroup $G$ of $S L_{2}(\mathbb{C})$. Via the McKay correspondence [Ma], there is a one-to-one correspondence between the finite subgroups of $S L_{2}(\mathbb{C})$ and the Dynkin diagrams of simply-laced types $A_{n}, D_{n}, E_{6}, E_{7}$ and $E_{8}$. Let us denote by $\mathfrak{g}$ the simple Lie algebra corresponding to $G$. From the exact correspondence we know that the rank of $\mathfrak{g}$ is $\left|G_{*}\right|-1$. 
The quotient $\mathbb{C}^{2} / G$ has an isolated simple singularity at 0 . There exists a minimal resolution $\widetilde{\mathbb{C}^{2} / G}$ of $\mathbb{C}^{2} / G$ well known as ALE-spaces (cf. e.g. $\mathbb{\mathbb { N }}$ ). It is known that the second homology group $H_{2}\left(\widetilde{\mathbb{C}^{2} / G}, \mathbb{Z}\right)$ is isomorphic to the root lattice of $\mathfrak{g}$, cf. e.g. $\mathbb{\mathbb { N }}$. In particular

$$
\operatorname{dim} K\left(\widetilde{\mathbb{C}^{2} / G}\right)=\operatorname{dim} H\left(\widetilde{\mathbb{C}^{2} / G}\right)=\left|G_{*}\right| .
$$

So if we apply Theorem 3 to the case $\widetilde{\mathbb{C}^{2} / G}$ with a trivial group action, we have

$$
\sum_{n \geq 0} \operatorname{dim} K_{S_{n}}\left({\widetilde{\mathbb{C}^{2} / G}}^{n}\right) q^{n}=\prod_{r \geq 1}\left(1-q^{r}\right)^{-\left|G_{*}\right|} .
$$

On the other hand, by the Thom isomorphism [S1] we have

$$
K_{G_{n}}\left(X^{n}\right) \approx K_{G_{n}}(p t)=R\left(G_{n}\right) .
$$

It follows from the part 3) of Remark 1 and Eq. (29) that

$$
\sum_{n \geq 0} \operatorname{dim} K_{G_{n}}\left(\mathbb{C}^{2 n}\right) q^{n}=\prod_{r \geq 1}\left(1-q^{r}\right)^{-\left|G_{*}\right|} .
$$

We can obtain another numerical coincidence from a somewhat different point of view as follows. For a general quasi-projective smooth surface $Y$, a well-known result of Fogarty says that the Hilbert scheme of $n$ points on $Y$, denoted by $Y^{[n]}$, is a smooth $2 n$ dimensional manifold. The Betti numbers of $Y^{[n]}$ were computed by Göttsche G]. In particular, Göttsche's formula yields the dimension of the homology group of $Y^{[n]}$ :

$$
\sum_{n \geq 0} \operatorname{dim} H\left(Y^{[n]}\right) q^{n}=\prod_{r \geq 1}\left(1-q^{r}\right)^{-\operatorname{dim} H(Y)} .
$$

We apply Eq. (30) to the case $Y=\widetilde{\mathbb{C}^{2} / G}$. It follows from Eq. (29) that

$$
\sum_{n \geq 0} \operatorname{dim} H\left(\widetilde{\mathbb{C}^{2} / G}{ }^{[n]}\right) q^{n}=\prod_{r \geq 1}\left(1-q^{r}\right)^{-\left|G_{*}\right|} .
$$

Therefore we have proved the following.

Proposition 5 The spaces $K_{G_{n}}\left(\mathbb{C}^{2 n}\right) \otimes \mathbb{C}, K_{S_{n}}\left({\widetilde{\mathbb{C}^{2} / G}}^{n}\right) \otimes \mathbb{C}$ and $H\left({\widetilde{\mathbb{C}^{2} / G}}^{[n]}\right)$ have the same dimension.

Since the minimal resolution $\widetilde{\mathbb{C}^{2} / G}$ of $\mathbb{C}^{2} / G$ has no odd dimensional homology, we have (cf. $[\mathrm{HH}, \mathbb{N}]$ )

$$
e\left(\widetilde{\mathbb{C}^{2} / G}\right)=\operatorname{dim} H\left(\widetilde{\mathbb{C}^{2} / G}\right)=e\left(\mathbb{C}^{2}, G\right)=\left|G_{*}\right| .
$$

The following corollary is a special and important case of Corollary 3 . 
Corollary 4 Let $G$ be a finite subgroup of $S L_{2}(\mathbb{C})$ and $\widetilde{\mathbb{C}^{2} / G}$ be the minimal resolution of $\mathbb{C}^{2} / G$. Then the Hilbert scheme of $n$ points on $\widetilde{\mathbb{C}^{2} / G}$ is a resolution of singularities of $\mathbb{C}^{2 n} / G_{n}$ whose Euler characteristic is equal to the orbifold Euler characteristic $e\left(\mathbb{C}^{2 n}, G_{n}\right)$.

Remark 6 The fact that the (graded) dimension of $K_{S_{n}}\left(X^{n}\right)$ equals that of the homology group of the Hilbert scheme of $n$ points of $X$ for a more general surface $X$ holds by the same argument as above. Bezrukavnikov and Ginzburg [BG] have proposed a way to establish a direct isomorphism between these two spaces for an algebraic surface $X$. M. de Cataldo and L. Migliorini has recently established an isomorphism independently for any complex surface [CM].

Proposition 5 can be generalized as follows. Assume that $X$ is a quasi-projective surface acted upon by $G$ and there exists a smooth resolution of singularities $\widetilde{X / G}$ of $X / G$ such that the dimension of $K_{G}^{i}(X)(i=0,1)$ equals that of the even (resp. odd) dimensional homology group of $X / G$. Then we conclude that the dimension of $K_{G_{n}}\left(X^{n}\right)$ is equal to that of the homology group of the Hilbert scheme of $n$ points on $\widetilde{X / G}$. We conjecture the existence of a natural isomorphism from $K_{G_{n}}\left(X^{n}\right) \otimes \mathbb{C}$ to $H\left(\widetilde{X / G}^{[n]}\right)$, assuming the (necessary) existence of an isomorphism from $K_{G}(X) \otimes \mathbb{C}$ to $H(\widetilde{X / G})$ or $K(\widetilde{X / G}) \otimes \mathbb{C}$.

We believe that this is just a first indication of intriguing relations between the Hilbert scheme of $n$ points on $\widetilde{X / G}$ and the wreath product $G_{n}$. We will elaborate on this in another occasion. When $G$ is trivial, it reduces to well-known relations between the Hilbert scheme of $n$ points and the symmetric group $S_{n}(\mathrm{cf} . \mathbb{\mathbb { N }}, \overline{\mathrm{BG}}]$ ).

Remark 7 Let us consider a special case of Corollary 4 by putting $G=\mathbb{Z}_{2}$. The wreath product $G_{n}$ in this case is exactly the Weyl group of $B_{n}$ or $C_{n}$. It is interesting to compare with a "Hilbert scheme" associated to a reductive group of type $B_{n}\left(\right.$ or $\left.C_{n}\right)$ defined in $\mathrm{BG}$.

Acknowledgement. The starting point of this work is the insight of Segal [S2]. I am grateful to him for his permission of using his unpublished results. I am also grateful to Igor Frenkel who first emphasized to me the importance of [52] and to Mikio Furuta whose generous help is indispensable for me to understand [S2] for inspiring and fruitful discussions. I thank Mark de Cataldo for helpful conversations on Hilbert schemes. I also thank the referee for his comments and suggestions which help to improve the presentation of the paper. This paper is a modified version of my MPI preprint "Equivariant K-theory and wreath products" in August 1998. It is a pleasure to acknowledge the warm hospitality of the MaxPlanck Institut für Mathematik at Bonn. 
Note 1 added. The idea here of relating the representation rings of wreath products associated to finite groups of $S L_{2}(\mathbb{C})$ and vertex representations of affine Lie algebras has been fully developed in a paper "Vertex representations via finite groups and the McKay correspondence" by I. Frenkel, N. Jing and the author.

Note 2 added. The connections among Hilbert schemes, wreath products and Ktheory have been developed in my recent paper "Hilbert schemes, wreath products, and the McKay correspondence".

\section{References}

[A] M. Atiyah, Power operations in K-theory, Quart. J. Math. Oxford 17 (1966) 165-193.

[A1] M. Atiyah, K-Theory, Benjamin, New York, 1967.

[AS] M. Atiyah and G. Segal, On equivariant Euler characteristics, J. Geom. Phys. 6 (1989) 671-677.

[BBM] P. Baum, J. Brylinski and R. MacPherson, Cohomologie équivariante délocalisée, C.R. Acad. Sci. Paris 300 (1985) 605-608.

[BC] P. Baum and A. Connes, Chern character for discrete groups, In: Y. Matsumoto et al (eds.), A Fete of Topology, Academic Press, 1988.

[BG] R. Bezrukavnikov and V. Ginzburg, Hilbert schemes and reductive groups, unpublished notes.

[B] R. Borcherds, Vertex algebras, Kac-Moody algebras, and the Monster, Proc. Natl. Acad. Sci, USA 83 (1986) 3068-3071.

[CM] M. de Cataldo and L. Migliorini, The Douady space of a complex surface, preprint, math.AG/9811159.

[DHVW] L. Dixon, J. Harvey, C. Vafa and E. Witten, Strings on orbifolds. I. Nucl. Phys. B 261 (1985) 678-686.

[FLM] I. Frenkel, J. Lepowsky and A. Meurman, Vertex operator algebras and the Monster, Academic Press, New York 1988.

[G] L. Göttsche, The Betti numbers of the Hilbert scheme of points on a smooth projective surface, Math. Ann. 286 (1990) 193-207.

[Gr] I. Grojnowski, Instantons and affine algebras I: the Hilbert scheme and vertex operators, Math. Res. Lett. 3 (1996) 275-291. 
[HH] F. Hirzebruch and T. Höfer, On the Euler number of an orbifold, Math. Ann. 286 (1990) 255-260.

[M] I. Macdonald, The Poincare polynomial of a symmetric product, Proc. Camb. Phil. Soc. 58 (1962) 563-568.

[M1] I. Macdonald, Polynomial functors and wreath products, J. Pure Appl. Algebra, 18 (1980) 173-204.

[M2] I. Macdonald, Symmetric Functions and Hall Polynomials, Second Edition, Oxford, Clarendon Press, 1995.

[Mc] J. McKay, Graphs, singularities and finite groups, Proc. Sympos. Pure Math. 37, AMS (1980) 183-186.

[N] H. Nakajima, Lectures on Hilbert schemes of points on surfaces, 1996, http://www.kusm,kyoto-u.ac.jp/ nakajima/TeX.html.

[N1] H. Nakajima, Heisenberg algebra and Hilbert schemes of points on projective surfaces, Ann. Math, 145 (1997) 379-388.

[S1] G. Segal, Equivariant K-theory, Publ. Math. IHES, 34 (1968) 129-151.

[S2] G. Segal, Equivariant K-theory and symmetric products, preprint 1996 (unpublished).

[Ser] J.-P. Serre, Linear representations of finite groups, Grad. Texts in Math. 42, Springer-Verlag.

[VW] C. Vafa and E. Witten, A strong coupling test of S-duality, Nucl. Phys. B 431 (1994) 3-77.

[Z] A. Zelevinsky, Representations of finite classical groups, Lect. Note in Math. 869, Springer-Verlag.

Department of Mathematics, Yale University, New Haven, CT 06520, USA

E-mail: wqwang@math.yale.edu 